\title{
Multidimensional study of hadronization in nuclei
}

\author{
The HERMES Collaboration
}

A. Airapetian ${ }^{12,15}$, N. Akopov ${ }^{26}$, Z. Akopov ${ }^{5}$, E.C. Aschenauer 6 ,a , W. Augustyniak ${ }^{25}$, R. Avakian ${ }^{26}$, A. Avetissian ${ }^{26}$, E. Avetisyan ${ }^{5}$, S. Belostotski ${ }^{18}$, N. Bianchi ${ }^{10}$, H.P. Blok ${ }^{17,24}$, A. Borissov ${ }^{5}$, J. Bowles ${ }^{13}$, I. Brodski ${ }^{12}$, V. Bryzgalov ${ }^{19}$, J. Burns $^{13}$, M. Capiluppi ${ }^{9}$, G.P. Capitani ${ }^{10}$, E. Cisbani $^{21}$, G. Ciullo ${ }^{9}$, M. Contalbrigo ${ }^{9}$, P.F. Dalpiaz ${ }^{9}$, W. Deconinck ${ }^{5}$, R. De Leo ${ }^{2}$, L. De Nardo ${ }^{11,5}$, E. De Sanctis ${ }^{10}$, M. Diefenthaler ${ }^{14,8}$, P. Di Nezza ${ }^{10}$, M. Düren ${ }^{12}$, M. Ehrenfried ${ }^{12}$, G. Elbakian $^{26}$, F. Ellinghaus ${ }^{4}$, R. Fabbri ${ }^{6}$, A. Fantoni ${ }^{10}$, L. Felawka ${ }^{22}$, S. Frullani ${ }^{21}$, G. Gapienko ${ }^{19}$, V. Gapienko ${ }^{19}$, G. Gavrilov ${ }^{5,18,22}$, V. Gharibyan ${ }^{26}$, F. Giordano ${ }^{5,9}$, S. Gliske ${ }^{15}$, M. Golembiovskaya ${ }^{6}$, L. Grigoryan ${ }^{26}$, C. Hadjidakis ${ }^{10}$, M. Hartig ${ }^{5}$, D. Hasch $^{10}$, A. Hillenbrand ${ }^{6}$, M. Hoek ${ }^{13}$, Y. Holler ${ }^{5}$, I. Hristova ${ }^{6}$, Y. Imazu ${ }^{23}$, A. Ivanilov ${ }^{19}$, H.E. Jackson ${ }^{1}$, H.S. Jo ${ }^{11}$, S. Joosten ${ }^{14}$, R. Kaiser ${ }^{13, b}$, G. Karyan ${ }^{26}$, T. Keri ${ }^{13,12}$, E. Kinney ${ }^{4}$, A. Kisselev ${ }^{18}$, N. Kobayashi ${ }^{23}$, V. Korotkov ${ }^{19}$, V. Kozlov ${ }^{16}$, P. Kravchenko ${ }^{8,18}$, V.G. Krivokhijine' ${ }^{7}$, L. Lagamba ${ }^{2}$, L. Lapikás ${ }^{17}$, I. Lehmann ${ }^{13}$, P. Lenisa ${ }^{9}$, A. López Ruiz ${ }^{11}$, W. Lorenzon ${ }^{15}$, X.-G. Lu ${ }^{6}$, X.-R. Lu ${ }^{23}$, B.-Q. Ma ${ }^{3}$, D. Mahon ${ }^{13}$, N.C.R. Makins ${ }^{14}$, S.I. Manaenkov ${ }^{18}$, L. Manfré21 ${ }^{21}$ Y. Mao ${ }^{3}$, B. Marianski ${ }^{25}$, A. Martinez de la Ossa ${ }^{5,4}$, H. Marukyan ${ }^{26}$, C.A. Miller ${ }^{22}$, Y. Miyachi ${ }^{23, \text { c }}$, A. Movsisyan ${ }^{26}$, V. Muccifora ${ }^{10}$, M. Murray ${ }^{13}$, A. Mussgiller ${ }^{5,8}$, E. Nappi ${ }^{2}$, Y. Naryshkin $^{18}$, A. Nass $^{8}$, M. Negodaev 6 , W.-D. Nowak ${ }^{6}$, L.L. Pappalardo ${ }^{9}$, R. Perez-Benito ${ }^{12}$, A. Petrosyan ${ }^{26}$, M. Raithel $^{8}$, P.E. Reimer ${ }^{1}$, A.R. Reolon ${ }^{10}$, C. Riedl ${ }^{6}$, K. Rith ${ }^{8}$, G. Rosner ${ }^{13}$, A. Rostomyan ${ }^{5}$, J. Rubin ${ }^{1,14}$, D. Ryckbosch ${ }^{11}$, Y. Salomatin ${ }^{19}$, F. Sanftl ${ }^{20,23}$, A. Schäfer ${ }^{20}$, G. Schnell ${ }^{6,11, d, e}$, B. Seitz ${ }^{13}$, T.-A. Shibata ${ }^{23}$, V. Shutov ${ }^{7}$, M. Stancari ${ }^{9}$, M. Statera ${ }^{9}$, E. Steffens ${ }^{8}$, J.J.M. Steijger ${ }^{17}$, J. Stewart ${ }^{6}$, F. Stinzing ${ }^{8}$, S. Taroian ${ }^{26}$, R. Truty ${ }^{14}$, A. Trzcinski ${ }^{25}$, M. Tytgat ${ }^{11}$, A. Vandenbroucke ${ }^{11}$, Y. Van Haarlem ${ }^{11}$, C. Van Hulse ${ }^{11}$, D. Veretennikov ${ }^{18}$, V. Vikhrov ${ }^{18}$, I. Vilardi ${ }^{2}$,

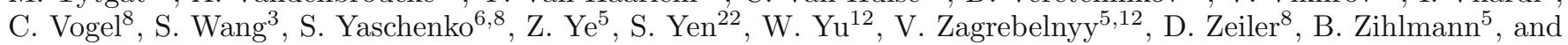
P. Zupranski ${ }^{25}$

\footnotetext{
1 Physics Division, Argonne National Laboratory, Argonne, Illinois 60439-4843, USA

2 Istituto Nazionale di Fisica Nucleare, Sezione di Bari, 70124 Bari, Italy

3 School of Physics, Peking University, Beijing 100871, China

4 Nuclear Physics Laboratory, University of Colorado, Boulder, Colorado 80309-0390, USA

5 DESY, 22603 Hamburg, Germany

6 DESY, 15738 Zeuthen, Germany

7 Joint Institute for Nuclear Research, 141980 Dubna, Russia

8 Physikalisches Institut, Universität Erlangen-Nürnberg, 91058 Erlangen, Germany

9 Istituto Nazionale di Fisica Nucleare, Sezione di Ferrara and Dipartimento di Fisica, Università di Ferrara, 44100 Ferrara, Italy

10 Istituto Nazionale di Fisica Nucleare, Laboratori Nazionali di Frascati, 00044 Frascati, Italy

11 Department of Subatomic and Radiation Physics, University of Gent, 9000 Gent, Belgium

12 Physikalisches Institut, Universität Gießen, 35392 Gießen, Germany

13 SUPA, School of Physics and Astronomy, University of Glasgow, Glasgow G12 8QQ, UK
}

\footnotetext{
a Now at: Brookhaven National Laboratory, Upton, New York 11772-5000, USA.

b Present address: International Atomic Energy Agency, A1400 Vienna, Austria.

c Now at: Department of Physics, Yamagata University Yamagata, 990-8560, Japan.

d Now at: Department of Theoretical Physics, University of the Basque Country UPV/EHU, 48080 Bilbao, and IKERBASQUE, Basque Foundation for Science, 48011 Bilbao, Spain.

e e-mail: gunar.schnell@desy.de
} 
14 Department of Physics, University of Illinois, Urbana, Illinois 61801-3080, USA

15 Randall Laboratory of Physics, University of Michigan, Ann Arbor, Michigan 48109-1040, USA

16 Lebedev Physical Institute, 117924 Moscow, Russia

17 National Institute for Subatomic Physics (Nikhef), 1009 DB Amsterdam, The Netherlands

18 Petersburg Nuclear Physics Institute, Gatchina, 188300 Leningrad Region, Russia

19 Institute for High Energy Physics, Protvino, 142281 Moscow Region, Russia

20 Institut für Theoretische Physik, Universität Regensburg, 93040 Regensburg, Germany

21 Istituto Nazionale di Fisica Nucleare, Sezione di Roma, Gruppo Collegato Sanità and Istituto Superiore di Sanità, 00161 Roma, Italy

${ }^{22}$ TRIUMF, Vancouver, British Columbia V6T 2A3, Canada

23 Department of Physics, Tokyo Institute of Technology, Tokyo 152, Japan

24 Department of Physics and Astronomy, VU University, 1081 HV Amsterdam, The Netherlands

25 National Centre for Nuclear Research, 00-689 Warsaw, Poland

26 Yerevan Physics Institute, 375036 Yerevan, Armenia

Received: 26 July 2011

Published online: 27 September 2011

(C) The Author(s) 2011. This article is published with open access at Springerlink.com

Communicated by Z.-E. Meziani

Abstract. Hadron multiplicities in semi-inclusive deep-inelastic scattering were measured on neon, krypton, and xenon targets relative to deuterium at an electron(positron)-beam energy of $27.6 \mathrm{GeV}$ at HERMES. These ratios were determined as a function of the virtual-photon energy $\nu$, its virtuality $Q^{2}$, the fractional hadron energy $z$ and the transverse hadron momentum with respect to the virtual-photon direction $p_{t}$. Dependences were analysed separately for positively and negatively charged pions and kaons as well as protons and antiprotons in a two-dimensional representation. Compared to the one-dimensional dependences, some new features were observed. In particular, when $z>0.4$ positive kaons do not show the strong monotonic rise of the multiplicity ratio with $\nu$ as exhibited by pions and negative kaons. Protons were found to behave very differently from the other hadrons. 


\section{Introduction}

The process of quark fragmentation and hadronization can be investigated by measuring hadron production in semiinclusive deep-inelastic scattering of leptons from nuclei of various sizes. As typical hadronization lengths are of the order of the size of a nucleus, the nuclei act as scale probes of the underlying hadronization mechanism, i.e., crosssections are expected to be sensitive to whether - in a semi-classical picture - the hadronization occurs within or outside the nucleus. Thus, the space (time) development of hadronization can be investigated. Such experiments were performed by the SLAC [1], EMC [2] and E665 [3] Collaborations. Recently, more precise data were collected and analysed by the HERMES [4-7] and CLAS [8] Collaborations. The HERMES and the preliminary CLAS data are complementary in that the latter cover values of the virtual-photon energy $\nu$ below $4 \mathrm{GeV}$. Compared to hadronic probes, the use of leptonic probes has the advantage that initial-state interactions can be neglected and that the energy and momentum transferred to the struck parton are well determined by the measured kinematic properties of the scattered lepton in the final state. The results of such studies of the hadronization in nuclei, i.e., cold nuclear matter, are expected to be useful for understanding the fundamental aspects of hadronization in the framework of quantum chromodynamics, as well as an input to the calculation of nuclear parton distribution functions (see, e.g., ref. [9]) and for the interpretation of jet-quenching and parton energy-loss phenomena in ultra-relativistic heavy-ion collisions (hot nuclear matter) [10-16].

The experimental data with leptonic probes are presented as the ratio of the hadron multiplicities observed in the scattering on a nucleus $(A)$ to those on the deuteron (D):

$$
R_{A}^{h}\left(\nu, Q^{2}, z, p_{t}^{2}\right)=\frac{\left(\frac{N^{h}\left(\nu, Q^{2}, z, p_{t}^{2}\right)}{N^{e}\left(\nu, Q^{2}\right)}\right)_{A}}{\left(\frac{N^{h}\left(\nu, Q^{2}, z, p_{t}^{2}\right)}{N^{e}\left(\nu, Q^{2}\right)}\right)_{D}}
$$

where $N^{h}$ is the number of semi-inclusive hadrons in a given $\left(\nu, Q^{2}, z, p_{t}^{2}\right)$ bin and $N^{e}$ the number of inclusive deep-inelastic scattering leptons in the same $\left(\nu, Q^{2}\right)$ bin. This ratio depends on leptonic variables: the energy $\nu$ of the virtual photon in the laboratory frame and its squared four-momentum $-Q^{2}$; and on hadronic variables: the fraction $z$ of the virtual-photon energy carried by the hadron and the square of the hadron momentum component $p_{t}^{2}$ transverse to the virtual-photon direction. In principle, the ratio also depends on the azimuthal angle $\phi$ between the lepton-scattering plane and the hadron-production plane. In the present measurement no dependence of $R_{A}^{h}$ on $\phi$ was observed within the statistical accuracy. As a consequence the integration over $\phi$ was performed.

In all previous publications results for $R_{A}^{h}$ were shown as a function of one variable only (one-dimensional dependences) except one case where a two-dimensional dependence was extracted for a combined sample of charged pions [7]. In the following, data for $R_{A}^{h}$ for neon (Ne), krypton $(\mathrm{Kr})$, and xenon (Xe) are presented in a twodimensional form for positively and negatively charged pions and kaons, and for protons and antiprotons separately. The two-dimensional representation consists in a fine binning in one variable and a coarser binning in another variable. The other variables are integrated over within the acceptance of the experiment. This allows the dependences to be studied in more detail, while keeping the statistical uncertainties at moderate levels, at least for pions, positive kaons and protons. Some of the most prominent features of the obtained results are presented and discussed. The full set of results is available in a database [17].

The wealth of theoretical model calculations and studies [18-35] reflect the strong interest of the community in hadron-multiplicities on nuclei, as they provide information on the space (time) structure of the hadronization process. It is beyond the scope of this paper to compare the results of the various models with the data. The onedimensional results published have already been instrumental in distinguishing between some models [7]. It is expected that the two-dimensional results presented here will further help discriminating between models.

\section{Experiment and data analysis}

The measurements were performed with the HERMES spectrometer [36] using $27.6 \mathrm{GeV}$ positron and electron beams stored in HERA at DESY. Data were collected during 1999, 2000, 2004 and 2005 with gaseous targets of deuterium, neon, krypton and xenon.

The experimental set-up and data analysis are described in detail in ref. [7]. Here, only a brief summary and update is given. The identification of charged hadrons was accomplished using information from the dual-radiator ring-imaging Cerenkov detector ( $\mathrm{RICH})$ [37], which provided separation of pions, kaons and (anti)protons in the momentum range between 2 and $15 \mathrm{GeV}$. Compared to the analysis described in ref. [7], an improved hadronidentification algorithm was used, which is based on a collective assignment of a set of identities to all particles detected in the event, accounting for the correlations among their probabilities $[38,39]$. The differences compared to the results obtained with the simpler approach neglecting such correlations were found to be minor and within the quoted systematic uncertainties for all particles.

The scattered leptons were selected using the following kinematic conditions: $\nu=4.0-23.5 \mathrm{GeV}$ (the upper bound corresponds to $y=\nu / E<0.85), Q^{2}>1 \mathrm{GeV}^{2}$, $W^{2}>4 \mathrm{GeV}^{2}$, where $E$ is the beam energy and $W$ is the invariant mass of the photon-nucleon system. The constraints on $y$ and $W^{2}$ were applied in order to limit the magnitude of radiative corrections and to suppress events originating from nucleon resonances, respectively. The kinematic constraints imposed on the selected hadrons were: $p_{h}=2-15 \mathrm{GeV}, z>0.2$ and $x_{F}>0$, where $p_{h}$ is the hadron momentum and the Feynman variable $x_{F}$ is defined as the ratio of the longitudinal momentum 

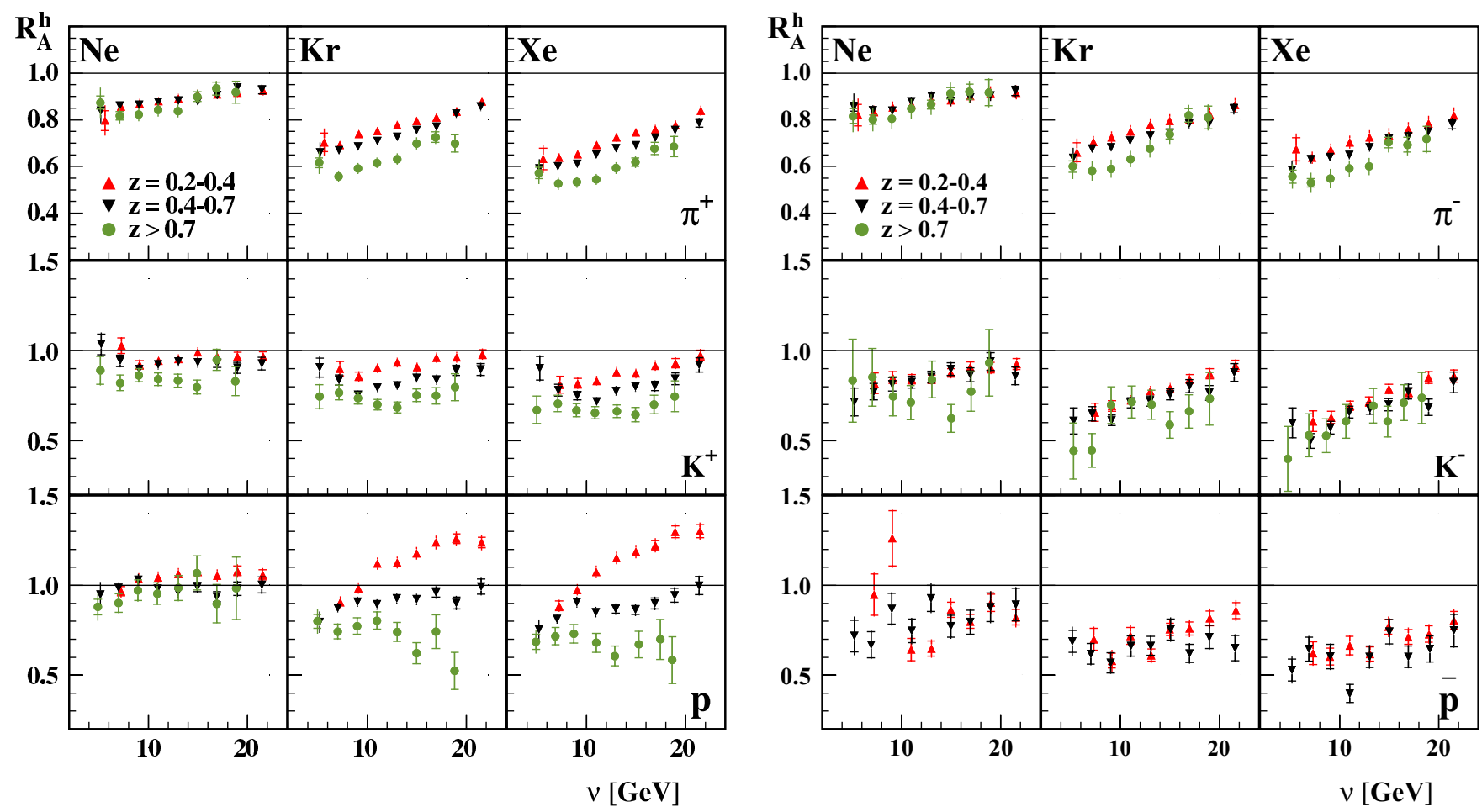

Fig. 1. Dependence of $R_{A}^{h}$ on $\nu$ for positively and negatively charged hadrons for three slices in $z$ as indicated in the legend. The inner and outer error bars indicate the statistical and total uncertainties, respectively. For the latter the statistical and systematic bin-to-bin uncertainties were added in quadrature. In addition, scale uncertainties of $3 \%, 5 \%, 4 \%$, and $10 \%$ are to be considered for pions, kaons, protons and antiprotons, respectively.

transferred to the hadron in the photon-nucleon centreof-mass system to its maximum possible value. Together, the constraints on $z$ and $x_{F}$ reduce contributions from the target fragmentation region.

From the data, the hadron multiplicity ratios $R_{A}^{h}$ were determined for each hadron type and target. Radiative corrections were applied following the scheme described in refs. [7,40-43], using average values of $\nu$ and $Q^{2}$ for each kinematic bin in the analysis. The corrections remain below $7 \%$ in all bins. Acceptance effects were studied in Monte Carlo simulations using an experimentally motivated parametrisation of $R_{A}^{h}$. They were found to be small compared to other uncertainties in all but the lowest bin in $\nu$. The differences between the parametrised and reconstructed values were used to estimate the systematic uncertainty due to the restricted acceptance for each hadron type.

Uncertainties in the knowledge of radiative processes (up to $2 \%$ ) and half of the observed maximal differences between results for $R_{A}^{h}$ from different data-taking periods were taken together as overall scale uncertainties ${ }^{1}$. The to-

\footnotetext{
${ }^{1}$ In order to reduce effects from statistical fluctuations larger ranges of acceptance were integrated for these studies. However, it was verified that those effects were not generated in certain kinematic ranges only.
}

tal scale uncertainties are $3 \%, 5 \%, 4 \%$, and $10 \%$ for pions, kaons, protons and antiprotons, respectively.

The uncertainties due to the hadron identification were estimated to be up to $0.5 \%$ for charged pions, up to $1.5 \%$ for kaons and protons, and up to $4 \%$ for antiprotons. Those due to acceptance effects were $6 \%$ for pions, $3 \%$ for kaons, and $7 \%$ for protons and antiprotons in the first $\nu$ bin, and less than $2 \%$ for any hadron in any other bin. Effects due to the contamination from diffractive $\rho^{0}$ meson production were estimated to be at most 4 and $7 \%$ for positive and negative pions, respectively. (For details see ref. [7].) These uncertainties were added in quadrature separately for each data point to yield systematic bin-to-bin uncertainties. Those were subsequently added in quadrature to the statistical uncertainties and plotted as total uncertainties.

\section{Results and discussion}

The results for the multiplicity ratio $R_{A}^{h}$ are presented using a fine binning in one of the variables, a coarser binning (called slice) in a second variable, and integrating over the remaining variables within the acceptance of the experiment. The following slices were used: $4-12,12-17$, and $17-23.5 \mathrm{GeV}$ for $\nu ; 0.2-0.4,0.4-0.7$, and $>0.7$ for $z$; and $\leq 0.4,0.4-0.7$, and $>0.7 \mathrm{GeV}^{2}$ in the case of $p_{t}^{2}$. The dependence on $Q^{2}$ was investigated, but as it turned out to 

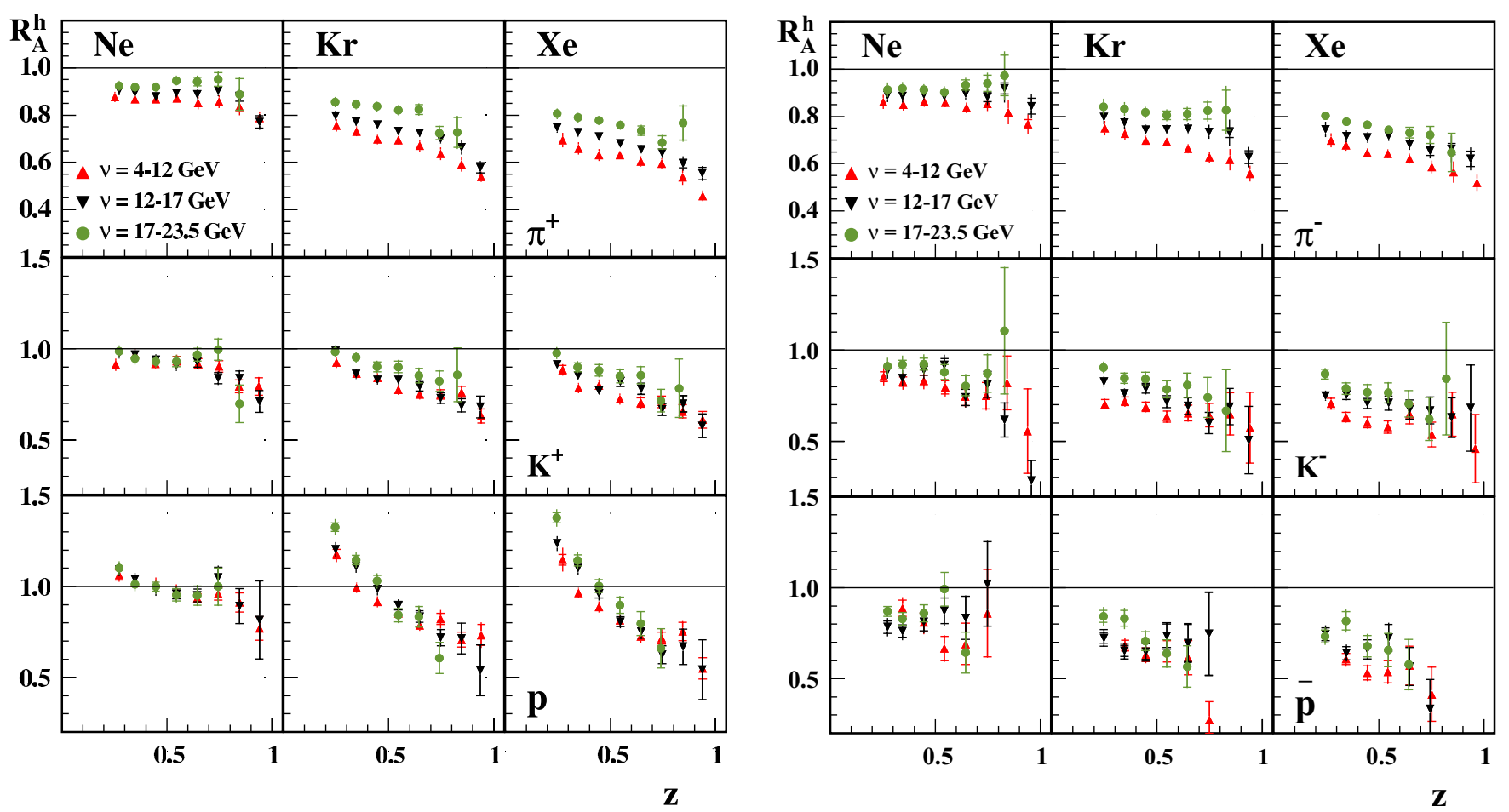

Fig. 2. Dependence of $R_{A}^{h}$ on $z$ for positively and negatively charged hadrons for three slices in $\nu$ as indicated in the legend. Uncertainties are shown as in fig. 1.

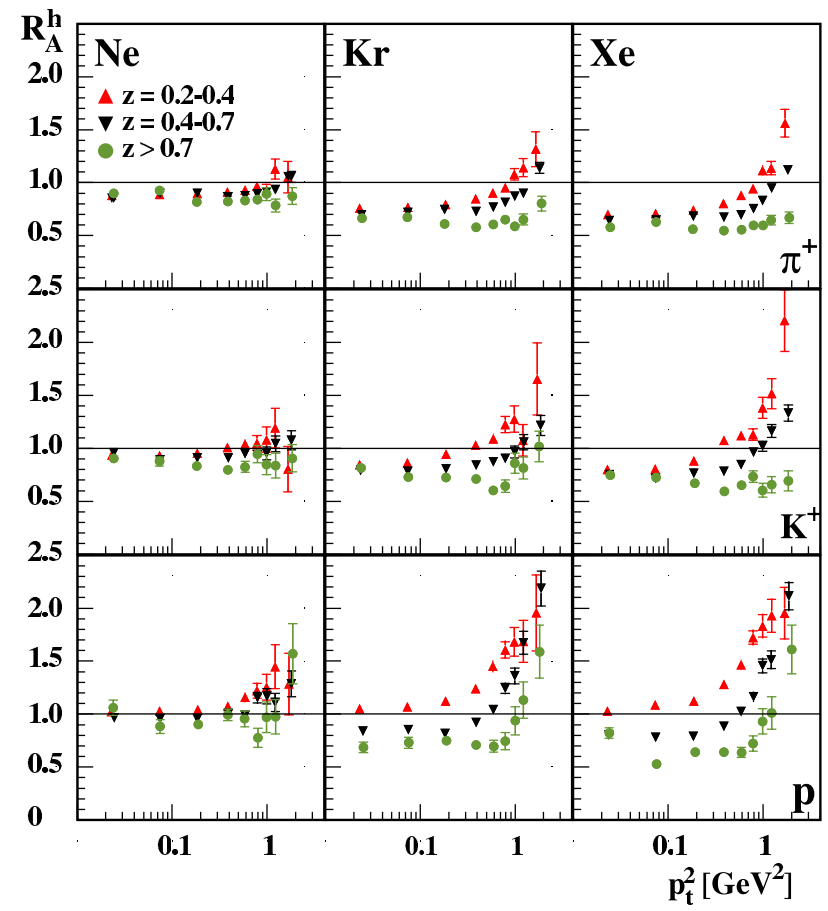

Fig. 3. Dependence of $R_{A}^{h}$ on $p_{t}^{2}$ for positively charged hadrons for three slices in $z$ as indicated in the legend. Uncertainties are shown as in fig. 1 .

be weak, no dependences with slices in $Q^{2}$ were produced. In the following, dependences that show salient features are discussed. In the presentation of the data, bins based on fewer than 10 events were omitted because the large statistical uncertainty would preclude useful conclusions.

The dependence of $R_{A}^{h}$ on $\nu$ for three slices in $z$ is shown in fig. 1. For pions and $\mathrm{K}^{-}$, a global trend of steady increase of $R_{A}^{h}$ with increasing values of $\nu$ was observed. Such a behaviour is explained in fragmentation models as resulting from Lorentz dilation and/or a shift in the argument $z$ of the relevant fragmentation function [18]. However, at the highest $z$ range there is an indication for a flattening out (and possibly a reversal of this trend) at low $\nu$ for $\pi^{+}$and $\pi^{-}$independently, which is not explained by these mechanisms.

The behaviour of $R_{A}^{h}$ for $\mathrm{K}^{+}$was found to be more complicated. For krypton and xenon there is a clear increase of $R_{A}^{\mathrm{K}^{+}}$with $\nu$ for the lowest $z$-slice, but at larger values of $z$ the behaviour is flatter. In contrast, the results for $R_{A}^{h}$ for $\mathrm{K}^{-}$resemble those for pions. For antiprotons, the $\nu$ dependence was found to be weak with a slightly positive slope, but the statistical accuracy of the results is too limited to draw definite conclusions. The neon data show similar but less pronounced trends, which was a common observation in all distributions under study. This is not unexpected due to the smaller size of the nucleus of neon compared to krypton and xenon.

The results for protons differ significantly from those for the other hadrons. For the heavy nuclei, $R_{A}^{\mathrm{p}}$ behaves very differently for the three $z$-slices, considerably exceeding unity at higher $\nu$ for the lowest $z$-slice. Part of the explanation may be the following. Unlike the other hadrons, protons are present already in the target nucleus. Therefore, apart from hadronization, residual protons can 

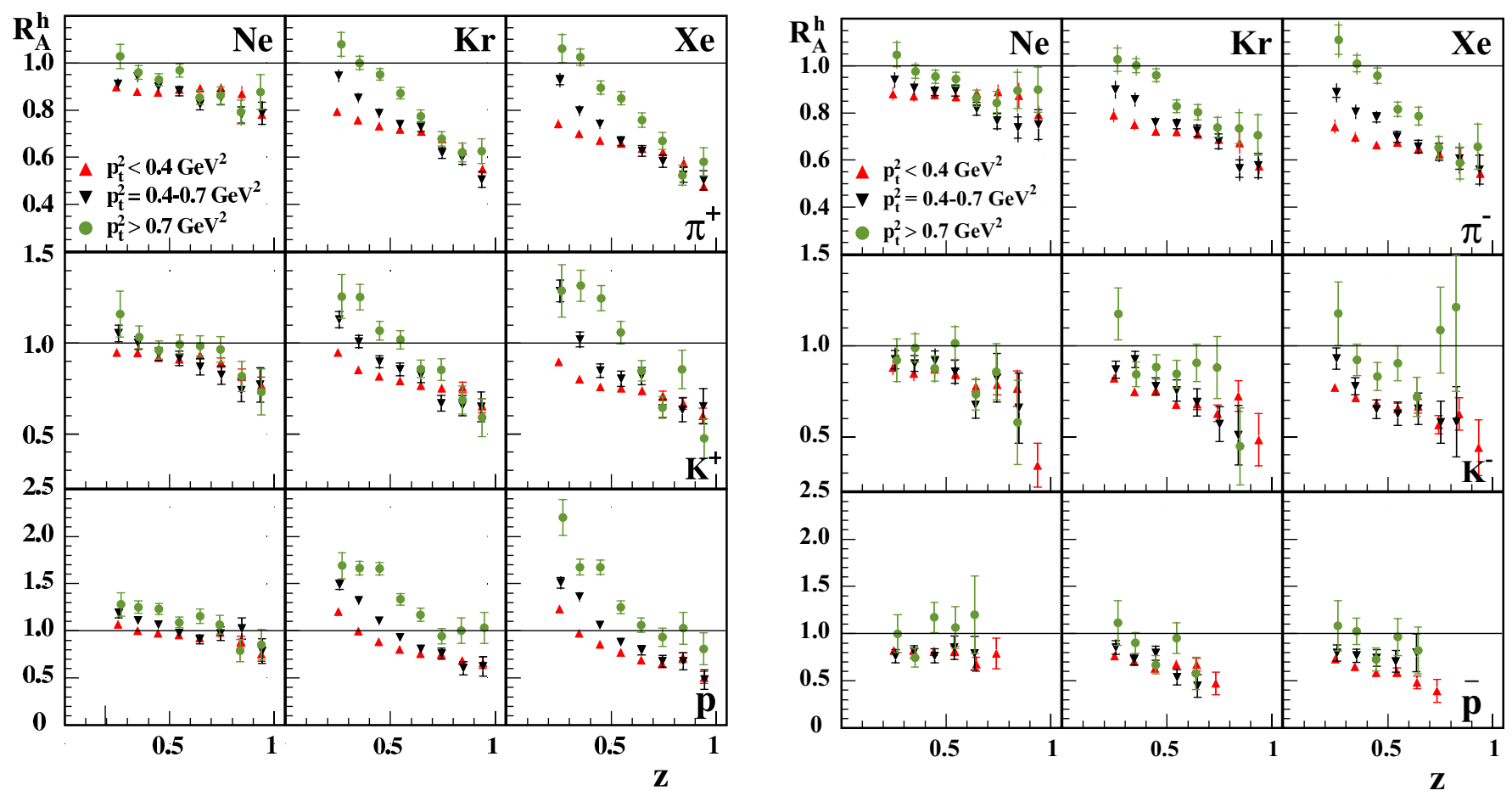

Fig. 4. Dependence of $R_{A}^{h}$ on $z$ for positively and negatively charged hadrons for three slices in $p_{t}^{2}$ as indicated in the legend. Uncertainties are shown as in fig. 1.

also result from reactions in the final state (final-state interactions), whereby a proton is knocked out of the nucleus. Those protons will preferably be emitted with low energy. This could lead to an energy dependence which, in conjunction with other kinematic factors, leads to the observed non-trivial behaviour.

The dependence of $R_{A}^{h}$ on $z$ for three ${ }^{2}$ slices in $\nu$ is shown in fig. 2. A slight change of the $z$ dependence when varying the $\nu$ range was observed for the $\pi^{+}$and $\pi^{-}$distributions. This has been observed already in ref. [7] for the combined pion sample and we refer to that paper for the discussion. The results on krypton and xenon for protons show a very strong dependence on $z$, the value of $R_{A}^{\mathrm{p}}$ exceeding unity in all $\nu$ ranges at low $z$. This supports the assumption that at low values of $z$ there is a sizable contribution of final-state interactions. A similar, but smaller effect was seen for $\mathrm{K}^{+}$, as $R_{A}^{\mathrm{K}^{+}}$increases to almost unity, while $R_{A}^{\mathrm{K}^{-}}$remains well below unity. This suggests that interactions play a role for $\mathrm{K}^{+}$production in which a proton in the target nucleus is transformed into a $\mathrm{K}^{+} \Lambda$ pair while the analogous process for $\mathrm{K}^{-}$ production is suppressed due to the quark content of the $\mathrm{K}^{-}[24]$.

Figure 3 shows the dependence of $R_{A}^{h}$ on $p_{t}^{2}$ for three slices in $z$ for positively charged hadrons. The behaviour of $R_{A}^{h}$ for $\pi^{-}$(not shown) was found to be the same as that for $\pi^{+}$within statistical uncertainties. The rise at high $p_{t}^{2}$

\footnotetext{
${ }^{2}$ As the combined dependence on $\nu$ and $z$ is crucial for various model calculations, the results as a function of $z$ are also given for five slices in $\nu$ in ref. [17].
}

suggests a broadening of the $p_{t}$ distribution [24]. Such a broadening could result from an interaction of the struck quark with the nuclear environment before the final hadron is produced and/or from interactions of the produced hadron within the nucleus. A detailed analysis and discussion of the HERMES data for pions and $\mathrm{K}^{+}$particles in terms of $p_{t}$ broadening has been presented in ref. [44]. Interesting to note is that in the highest $z$-slice $R_{A}^{h}$ for pions and $\mathrm{K}^{+}$becomes independent of $p_{t}^{2}$ within statistical uncertainties, while for protons a significant rise is observed at high $p_{t}^{2}$. For $\mathrm{K}^{-}$and antiprotons (neither are shown) limited statistics preclude any definite conclusion. In the intermediate $z$-range protons also show a much stronger rise with $p_{t}^{2}$ compared to pions and kaons in the respective ranges. This is consistent with a large contribution of final-state interactions in the case of protons.

In fig. 4 , the variation of the $p_{t}^{2}$-dependence with $z$ is presented in a different way by showing the dependence of $R_{A}^{h}$ on $z$ for three slices in $p_{t}^{2}$. The global decrease of $R_{A}^{h}$ with $z$ was already observed in fig. 2 . This dependence of $R_{A}^{h}$ on $z$ turns out to be stronger at higher values of $p_{t}^{2}$, an effect that is emphasised at larger target mass. At high $z$, the dependence on $p_{t}^{2}$ disappears for $\pi^{+}, \pi^{-}$, and $\mathrm{K}^{+}$, as has already been seen in fig. 3 . This lack of nuclear broadening of the $p_{t}$ distribution in the limit of instantaneous hadronization, i.e., before the struck parton has lost any energy, has been interpreted in terms of broadening arising from partonic processes [24]. For protons, a similar, but much stronger dependence of the slope on $p_{t}^{2}$ was observed, with $R_{A}^{\mathrm{p}}$ increasing far above unity at low $z$. This has been discussed in relation to fig. 2 as be- 
ing an indication of final-state interactions. The fact that the values of $R_{A}^{h}$ for $\mathrm{K}^{+}$are in between those for pions and protons suggests that, in addition to fragmentation, again final-state interactions play a role here. The large uncertainties of $R_{A}^{h}$ for $\mathrm{K}^{-}$and antiprotons preclude any particular conclusion in those cases.

\section{Conclusions}

Two-dimensional kinematic dependences have been presented for the multiplicity ratio $R_{A}^{h}$ for identified $\pi^{+}$, $\pi^{-}, \mathrm{K}^{+}, \mathrm{K}^{-}$, protons and antiprotons, measured in semi-inclusive deep-inelastic scattering of electrons and positrons from either neon, krypton, or xenon relative to deuterium. These two-dimensional distributions provide detailed information, which in some cases is not accessible in the one-dimensional distributions (in which all kinematic variables except one are integrated over, as has been traditionally done).

The behaviour of $R_{A}^{h}$ for $\pi^{+}$and $\pi^{-}$was found to be the same within the experimental uncertainties and is globally characterised by an increase of $R_{A}^{h}$ with the total energy transfer $\nu$ and a decrease with the fractional energy $z$ of the produced hadron. Negatively charged kaons behave similarly to pions, while the dependence of $R_{A}^{\mathrm{K}^{+}}$for positively charged kaons on $\nu$ changes depending on the value of $z$, possibly due to final-state interactions. Protons behave very differently from the other hadrons, especially in the $\nu$ distribution for different values of $z$. This may be explained by a sizable contribution of final-state interactions, such as knock-out processes, in addition to the fragmentation process. These new detailed data are expected to be an essential ingredient for constraining models of hadronization and, hence, improving our understanding of hadron formation.

We gratefully acknowledge the DESY management for its support and the staff at DESY and the collaborating institutions for their significant effort. This work was supported by: the Ministry of Economy and the Ministry of Education and Science of Armenia; the FWO-Flanders and IWT, Belgium; the Natural Sciences and Engineering Research Council of Canada; the National Natural Science Foundation of China; the Alexander von Humboldt Stiftung; the German Bundesministerium für Bildung und Forschung (BMBF); the Deutsche Forschungsgemeinschaft (DFG); the Italian Istituto Nazionale di Fisica Nucleare (INFN); the MEXT, JSPS, and G-COE of Japan; the Dutch Foundation for Fundamenteel Onderzoek der Materie (FOM); the Russian Academy of Science and the Russian Federal Agency for Science and Innovations; the U.K. Engineering and Physical Sciences Research Council, the Science and Technology Facilities Council, and the Scottish Universities Physics Alliance; the Basque Foundation for Science (IKERBASQUE); the U.S. Department of Energy (DOE) and the National Science Foundation (NSF); and the European Community Research Infrastructure Integrating Activity under the FP7 "Study of strongly interacting matter" (HadronPhysics2, Grant Agreement number 227431).
Open Access This article is distributed under the terms of the Creative Commons Attribution Noncommercial License which permits any noncommercial use, distribution, and reproduction in any medium, provided the original author(s) and source are credited.

\section{References}

1. L. Osborne et al., Phys. Rev. Lett. 40, 1624 (1978).

2. EMC Collaboration (J. Ashman et al.), Z. Phys. C 52, 1 (1991).

3. E665 Collaboration (M. Adams et al.), Phys. Rev. D 50, 1836 (1994).

4. HERMES Collaboration (A. Airapetian et al.), Eur. Phys. J. C 20, 479 (2001).

5. HERMES Collaboration (A. Airapetian et al.), Phys. Lett. B 577, 37 (2003).

6. HERMES Collaboration (A. Airapetian et al.), Phys. Rev. Lett. 96, 162301 (2006).

7. HERMES Collaboration (A. Airapetian et al.), Nucl. Phys. B 780, 1 (2007).

8. CLAS Collaboration (H. Hakobyan et al.), AIP Conf. Proc. 1265, 230 (2010).

9. K.J. Eskola et al., JHEP 04, 065 (2009).

10. PHENIX Collaboration (K. Adcox et al.), Phys. Rev. Lett. 88, 242301 (2002).

11. PHENIX Collaboration (K. Adcox et al.), Nucl. Phys. A 757, 184 (2005).

12. STAR Collaboration (J. Adams et al.), Phys. Rev. Lett. 92, 112301 (2004).

13. STAR Collaboration (J. Adams et al.), Phys. Lett. B 637, 161 (2006).

14. ATLAS Collaboration (G. Aad et al.), Phys. Rev. Lett. 105, 252303 (2010).

15. CMS Collaboration (S. Chatrchyan et al.), JHEP 07, 076 (2011).

16. ALICE Collaboration (K. Aamodt et al.), Phys. Lett. B 696, 30 (2011).

17. Durham HEP database, http://durpdg.dur.ac.uk; inSPIRE, http://inspirebeta.net/record/918944/; mailto: management@hermes.desy.de.

18. A. Accardi et al., Riv. Nuovo Cimento 32, 439 (2010).

19. A. Bialas, Acta Phys. Pol. B 11, 475 (1980).

20. A. Bialas, M. Gyulassy, Nucl. Phys. B 291, 793 (1987).

21. J. Czyzewski, P. Sawicki, Z. Phys. C 56, 493 (1992).

22. A. Accardi, V. Muccifora, H.J. Pirner, Nucl. Phys. A 720, 131 (2003).

23. A. Accardi, Phys. Lett. B 649, 384 (2007).

24. B.Z. Kopeliovich et al., Nucl. Phys. A 740, 211 (2004).

25. T. Falter et al., Phys. Lett. B 594, 61 (2004).

26. T. Falter et al., Phys. Rev. C 70, 054609 (2004).

27. N. Akopov, G. Elbakian, L. Grigoryan, hep-ph/0205123 (2002).

28. N. Akopov, L. Grigoryan, Z. Akopov, Eur. Phys. J. C 44, 219 (2005).

29. N. Akopov, L. Grigoryan, Z. Akopov, Phys. Rev. C 76, 065203 (2007).

30. X. Guo, X.-N. Wang, Phys. Rev. Lett. 85, 3591 (2000).

31. X.-N. Wang, X. Guo, Nucl. Phys. A 696, 788 (2001).

32. E. Wang, X.-N. Wang, Phys. Rev. Lett. 89, 162301 (2002).

33. F. Arleo, Eur. Phys. J. C 30, 213 (2003).

34. K. Gallmeister, U. Mosel, Nucl. Phys. A 801, 68 (2008). 
35. R. Sassot et al., Phys. Rev. D 81, 054001 (2010).

36. HERMES Collaboration (K. Ackerstaff et al.), Nucl. Instrum. Methods A 417, 230 (1998).

37. N. Akopov et al., Nucl. Instrum. Methods A 479, 511 (2002).

38. R. Lamb, PhD thesis, University of Illinois, August 2010, DESY-THESIS-2010-035.

39. HERMES Collaboration (E. Cisbani), Nucl. Phys. B 78, 366 (1999).
40. A.A. Akhundov, D. Yu Bardin, N.M. Shumeiko, Sov. J. Nucl. Phys. 26, 660 (1977).

41. D. Yu Bardin, N.M. Shumeiko, Sov. J. Nucl. Phys. 29, 499 (1979).

42. A.A. Akhundov et al., Sov. J. Nucl. Phys. 44, 988 (1986).

43. I. Akushevich, N. Shumeiko, A. Soroko, Eur. Phys. J. C 10, 681 (1999).

44. HERMES Collaboration (A. Airapetian et al.), Phys. Lett. B 684, 114 (2010). 